\title{
Correlated cryogenic fluorescence microscopy and electron cryo-tomography shows that exogenous TRIM5 $\alpha$ can form hexagonal lattices or autophagy aggregates in vivo
}

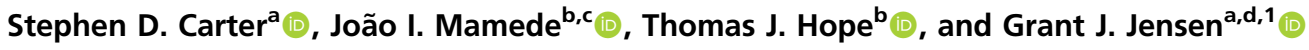 \\ ${ }^{a}$ Division of Biology and Biological Engineering, California Institute of Technology, Pasadena, CA 91125; ${ }^{b}$ Department of Cell and Developmental Biology, \\ Northwestern University, Chicago, IL 60611; 'Department of Microbial Pathogens and Immunity, Rush University Medical Center, Chicago, IL 60612; \\ and ${ }^{\mathrm{d}}$ HHMI, California Institute of Technology, Pasadena, CA 91125
}

Edited by John M. Coffin, Tufts University, Boston, MA, and approved September 23, 2020 (received for review November 19, 2019)

Members of the tripartite motif (TRIM) protein family have been shown to assemble into structures in both the nucleus and cytoplasm. One TRIM protein family member, TRIM5 $\alpha$, has been shown to form cytoplasmic bodies involved in restricting retroviruses such as HIV-1. Here we applied cryogenic correlated light and electron microscopy, combined with electron cryo-tomography, to intact mammalian cells expressing YFP-rhTRIM5 $\alpha$ and found the presence of hexagonal nets whose arm lengths were similar to those of the hexagonal nets formed by purified TRIM5 $\alpha$ in vitro. We also observed YFP-rhTRIM5 $\alpha$ within a diversity of structures with characteristics expected for organelles involved in different stages of macroautophagy, including disorganized protein aggregations (sequestosomes), sequestosomes flanked by flat double-membraned vesicles (sequestosome:phagophore complexes), sequestosomes within double-membraned vesicles (autophagosomes), and sequestosomes within multivesicular autophagic vacuoles (amphisomes or autolysosomes). Vaults were also seen in these structures, consistent with their role in autophagy. Our data 1) support recent reports that TRIM5 $\alpha$ can form both well-organized signaling complexes and nonsignaling aggregates, 2) offer images of the macroautophagy pathway in a near-native state, and 3) reveal that vaults arrive early in macroautophagy.

cryo-CLEM/ECT | TRIM5 $\alpha$-body | hexagonal nets | autophagy |

endoplasmic reticulum

$\mathbf{M}$ embers of the tripartite motif (TRIM) family of proteins have been shown to localize to isolated subcellular compartments (1). One of the most widely studied TRIM compartments is the TRIM5 $\alpha$ cytoplasmic body (2). Similar to other members of the TRIM family (1), TRIM5 proteins comprise a RING E3 ubiquitin-ligase domain, a B-box 2 self-assembly domain, and an antiparallel dimeric coiled-coil domain. Previous structural studies have shown that the coiled-coil and B-box 2 domains of TRIM5 $\alpha$ facilitate oligomerization into flat hexagonal lattices in vitro (3-6). TRIM5 proteins also contain one of two different C-terminal viral recognition domains, a B30.2/ SPRY domain in TRIM5 $\alpha$ or a cyclophilin A (CypA) domain in TRIMCyp (7-10). One of the most notable features of TRIM5 proteins is their ability to block retroviral infections by binding viral capsids via their B30.2/SPRY or CypA domains $(2,10)$, but how this leads to restriction remains unclear.

Campbell et al. reported previously that fluorescent-tagged human TRIM5 $\alpha$ (huTRIM5 $\alpha$ ) and rhesus TRIM5 $\alpha(\operatorname{rhTRIM} 5 \alpha)$ form highly dynamic fluorescent bodies of various sizes inside cells $(11,12)$, which interact with cytoplasmic HIV-1 viral complexes (12). Previous focused ion beam scanning electron microscopy studies revealed that fixed and stained YFP-tagged rhesus TRIM5 $\alpha$ (YFP-rhTRIM5 $\alpha$ ) bodies appear as large aggregates inside the cytoplasm (13), but no further details were discernible.
Toward elucidating the ultrastructure of YFP-rhTRIM5 $\alpha$ bodies to higher resolution and in a more native state, here we sought to image them using cryogenic correlated light and electron microscopy (cryo-CLEM), combined with electron cryo-tomography (cryo-ET) (14).

We find that YFP-rhTRIM5 $\alpha$ can form extended hexagonal nets inside cells, as well as different structures whose features match those expected for different stages of macroautophagy (15), including sequestosomes (16), phagophores, autophagosomes, and autophagic vacuoles. Macroautophagy is one form of autophagy in which large structures, such as mitochondria, misfolded and aggregated proteins, viruses, or other organelles or cellular pathogens, are degraded $(17,18)$. The process begins with sequestration of the substrates within the cytoplasm. Then a cup-shaped, flattened vesicle called a phagophore grows around the sequestered material until it fuses with itself, enclosing the substrates within a double-membraned autophagosome. Autophagosomes then travel long distances along microtubules toward the microtubule-organizing center until they encounter and fuse with either an endosome or a lysosome to form an amphisome or autolysosome, respectively. Membrane fusion of an amphisome with a lysosome can also produce an autolysosome.

\section{Significance}

One of the most notable features of TRIM5 proteins is their ability to restrict retroviral infections by binding viral capsids. TRIM5 $\alpha$ forms highly dynamic puncta of various sizes, and, when purified, hexagonal nets on the surface of HIV virions, but the molecular ultrastructure of the cellular bodies and the relationship of the in vitro nets to HIV restriction has remained unclear. To define the cellular ultrastructure underlying the punctate and dynamic nature of YFP-rhTRIM5 $\alpha$ bodies, we applied cryogenic correlated light and electron microscopy combined with electron cryo-tomography to TRIM5 $\alpha$ bodies and observed YFP-rhTRIM5 $\alpha$-localization to organelles found along the aggrephagy branch of the autophagy pathway. Consistent with previous work, we also found that TRIM5 $\alpha$ forms hexagonal nets inside cells.

Author contributions: S.D.C., J.I.M., T.J.H., and G.J.J. designed research; S.D.C. and J.I.M. performed research; S.D.C., J.I.M., T.J.H., and G.J.J. contributed new reagents/analytic tools; S.D.C., J.I.M., T.J.H., and G.J.J. analyzed data; and S.D.C. and G.J.J. wrote the paper. The authors declare no competing interest.

This article is a PNAS Direct Submission.

This open access article is distributed under Creative Commons Attribution-NonCommercialNoDerivatives License 4.0 (CC BY-NC-ND).

${ }^{1}$ To whom correspondence may be addressed. Email: jensen@caltech.edu.

This article contains supporting information online at https://www.pnas.org/lookup/suppl/ doi:10.1073/pnas.1920323117/-/DCSupplemental.

First published November 5, 2020. 
Another structure known to be involved in autophagy are vaults. Vaults are $70 \mathrm{~nm} \times 30 \mathrm{~nm}$, roughly ellipsoidal, megadalton ribonucleoprotein complexes comprising the major vault protein (MVP), telomerase-associated protein-1 (TEP1), and the vault poly (ADP ribose) polymerase. Vaults reside in the cytoplasm (19) and nucleus and are known to house noncoding vault RNAs important in the regulation of autophagy (20).

As a result of finding that YFP-rhTRIM5 $\alpha$ bodies are associated with macroautophagy, our cryo-tomograms provide threedimensional (3D) images of all these structures in their native state to macromolecular $(\sim 4 \mathrm{~nm})$ resolution and reveal that vaults arrive early in the process.

\section{Results}

HeLa cells either stably overexpressing YFP-rhTRIM5 $\alpha$ or not (wild-type controls) were grown on cryo-EM grids for $12 \mathrm{~h}$ and then plunge-frozen in liquid propane/ethane (21). Because the addition of the proteasome inhibitor MG-132 results in the formation of large YFP-rhTRIM5 $\alpha$ fluorescent bodies and has little effect on steady-state protein levels of YFP-rhTRIM5 $\alpha$ (compared with untreated) $(12,22)$, cells treated with MG-132 were also imaged. (SI Appendix, Table S2 provides details on which cryo-tomograms came from which cells.) Phase-contrast and fluorescence images of the frozen cells were then recorded using a light microscope equipped with a cryo-stage and a long working distance air-objective lens $(14,23)$. Grids with YFPrhTRIM5 $\alpha$ fluorescent bodies present in cell peripheries suitably thin for transmission electron microscopy were then transferred into a cryo-electron microscope. These same cell regions were then relocated within the cryo-electron microscope, and full tilt-series were recorded with and without the Volta phase plate (VPP). Then 3D reconstructions (i.e., cryo-tomograms) were calculated. Blue fluorospheres $(500 \mathrm{~nm})$ added to the samples before freezing were used as fiducial markers to precisely superimpose the centroids of the fluorescent puncta with $80-\mathrm{nm}$ accuracy onto the cryo-tomograms, using the same image correlation procedures described previously (24) (SI Appendix, Fig. S2).

A total of 45 cryo-tomograms ( 9 collected with the VPP) of YFP-rhTRIM5 $\alpha$ fluorescent bodies located in the cell periphery of 20 cells and 51 control cryo-tomograms of HeLa cells not expressing YFP-rhTRIM5 $\alpha$ were analyzed. As we inspected the cryo-tomograms of YFP-rhTRIM5 $\alpha$ bodies and compared them with the controls, we recognized several structures frequently colocalizing with YFP-rhTRIM5 $\alpha$, including vaults, dense protein aggregates, flattened vesicles, double-membraned vesicles, and nested vesicles. Because all these structures are expected for the autophagy pathway, we concluded that exogenous YFPrhTRIM $5 \alpha$ was being degraded through autophagy. This allowed us to begin to place the cryo-tomograms in sequence within the autophagy process based on which features accompanied which others (protein aggregates were sometimes present without flattened vesicles, and vice-versa) and also on existing knowledge, including, for instance, that phagophores (the flattened vesicles) are known to mature into double-membraned autophagosomes. This analysis revealed distinct stages of autophagy and their order: colocalization of substrate, arrival of vaults, formation of dense substrate aggregates (sequestosomes), creation of phagophores, phagophore closure around substrate to produce autophagosomes, and finally autophagosome fusion with either endosomes or lysosomes to produce an autophagic vacuole.

To confirm that the YFP-rhTRIM5 $\alpha$ bodies were in fact autophagic structures, we used immunofluorescence microscopy on cells grown in the same conditions (on cryo-EM grids) to check how often YFP-rhTRIM5 $\alpha$ bodies colocalized with two known autophagic markers, p62 and LC3B, as well as with two control nonautophagic markers, DCP1A and RAB11 (Fig. 1 and SI Appendix, Fig. S1). We found that $65 \%$ of YFP-rhTRIM5 $\alpha$ bodies colocalized with p62 puncta and 50\% colocalized with LC3B puncta. This compares well with the cryo-tomography findings, in which $71 \%$ of YFP-rhTRIM5a bodies localized to structures that we interpreted to be p62 sequestosomes (16) and $44 \%$ of YFP-rhTRIM5a bodies localized to structures that we interpreted to be stages of autophagy in which LC3B would be expected to reside, including phagophores, autophagosomes, and lysosomes (25).

In the figures, we present example cryo-tomograms representing each of the different stages of autophagy in their deduced sequence. For clarity and simplicity, each such figure shows one cryo-tomogram, with multiple panels to highlight different features: Fig. 2 and SI Appendix, Fig. S3 show vaults, Fig. 3 and SI Appendix, Figs. S4-S6 show prephagophore sequestosomes. Fig. 4 and SI Appendix, Figs. S7 and S8 show sequestosome:phagophore complexes, Fig. 5 and SI Appendix, Figs. S10 and S11 show autophagosomes, and Fig. 6 and SI Appendix, Fig. S12 show autophagic vacuoles. Again for clarity and simplicity, these figure panels follow a consistent pattern: the first panel shows an overlay of the yellowchannel fluorescence image (revealing the location of YFP-rhTRIM5 $\alpha$ ) on an $x y$ slice of the cryo-tomogram, the second panel shows the same slice of the cryo-tomogram without the fluorescence, the third panel shows a color-coded segmentation of the 3D cellular ultrastructure as revealed by the cryo-tomogram, and the fourth panel shows an enlarged view of key structures of interest, sometimes rotated to a more useful perspective. In some cases, additional panels or figures are shown to highlight other important findings. Two movies showing the correlation process, with cryo-tomograms and 3D segmentations of the events shown in Fig. 3 and SI Appendix, Figs. S6 and S9 and Fig. 7, are provided as Movies S1 and S2, respectively. We have also included nine additional movies to support Fig. 4 (Movies S3 and S4), Fig. 5 (Movie S5), Fig. 6 (Movie S6), SI Appendix, Fig. S5 (Movie S7), SI Appendix, Fig. S7 (Movie S8), SI Appendix, Fig. S8 (Movie S9), SI Appendix, Fig. S10 (Movie S10), SI Appendix, Fig. S11 (Movie S11). The prefigure in Fig. 3 illustrates the color code used in the segmentations to mark the different organelles.

Stage 1: Colocalization of Substrate. In 5 of the 45 cryo-tomograms of YFP-rhTRIM5 $\alpha$ fluorescent bodies, none of the aforementioned hallmarks of autophagy were found, and the scene was just typical cytoplasm (as in the controls). Our interpretation of this finding is that autophagy begins by substrate colocalization that is sufficient to form a fluorescent punctum but without structural features recognizable by cryo-ET.

Stage 2: Vault Complexes. Vaults, with their unique and readily recognizable morphology, were seen in 8 of the 45 total cryotomograms. Because no vaults were seen in the control cryotomograms of HeLa cells not expressing YFP-rhTRIM5 $\alpha$, we concluded that vaults are hallmarks of the autophagy pathway, rather than just ubiquitously present in the cytoplasm. Vaults were seen in cryo-tomograms alone (without any other hallmarks of autophagy visible) and at all other stages (with sequestosomes, phagophores, and autophagosomes and within autophagic vacuoles). Given the highly significant finding of vaults appearing alone in two cases (shown in Fig. 2 and SI Appendix, Fig. S3) and not in control cryo-tomograms but also in all other stages of autophagy $(P<0.004)$, we interpret these two cryo-tomograms as reflecting early stages of autophagy and conclude that vaults are recruited before the formation of visible sequestosomes.

Stage 3: Prephagophore Sequestosomes. Eighteen of the 45 YFPrhTRIM5 $\alpha$ fluorescent bodies colocalized with disorganized protein aggregates in the midst of unstructured cytoplasm that were recognizable both from their unique texture of packed dotlike densities and from their exclusion of organelles and vesicles. We applied a convolution neural network to objectively identify 
A

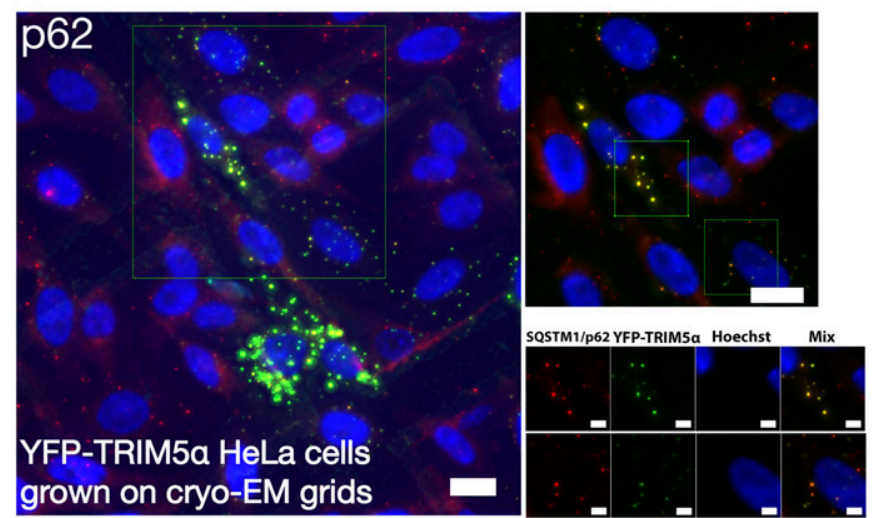

C

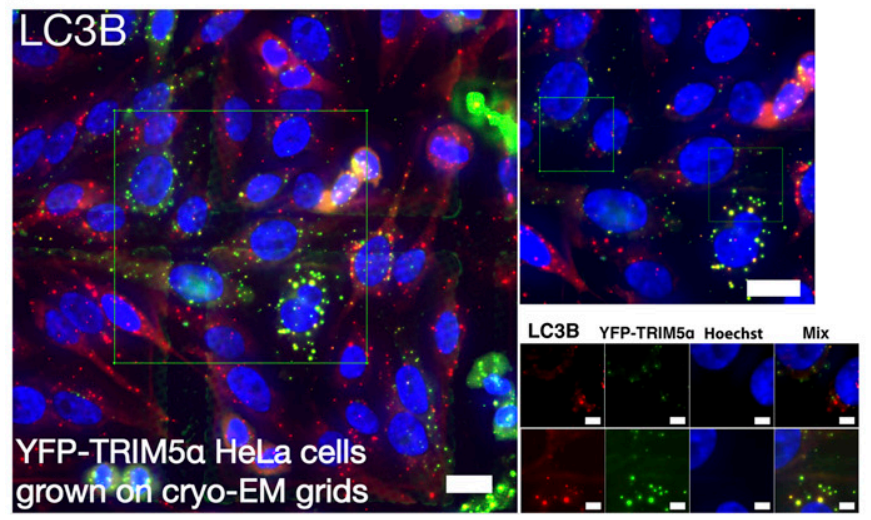

B

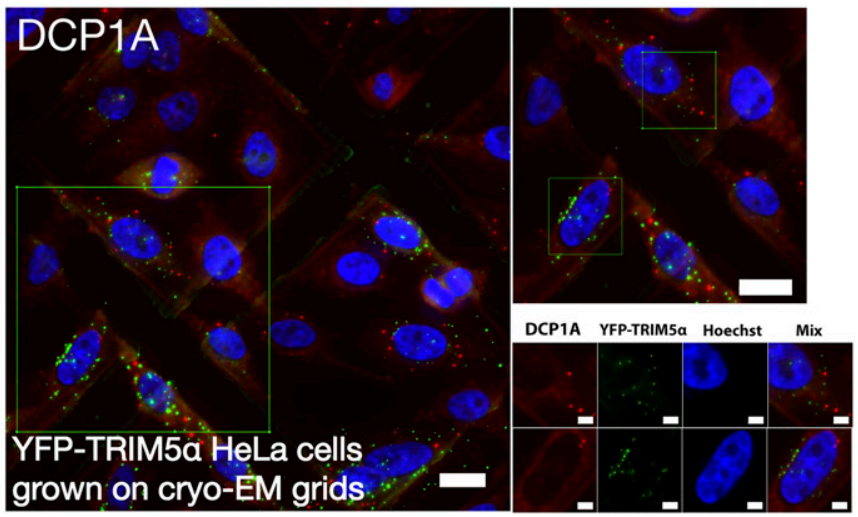

D

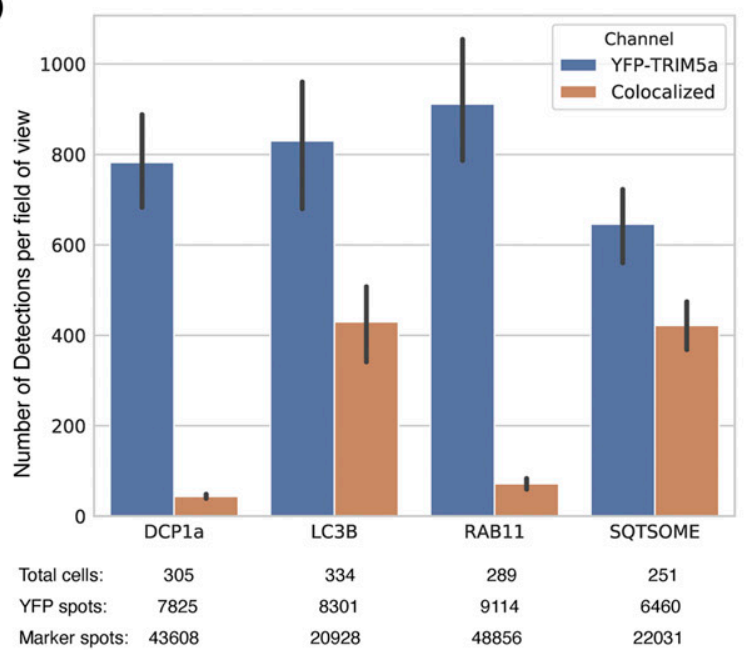

Fig. 1. YFP-rhTRIM5 $\alpha$ colocalizes with known autophagy markers in HeLa cells grown on EM grids. $(A-C)$ Total fields of view and subsets showing colocalization of YFP-rhTRIM5 $\alpha$ and SQSTM1/p62 (A) and LC3B $(B)$ but not of a control protein, DCP1A (C) (images of a similar control, RAB11, were comparable to DCP1A). Images showing the full field of view have the same contrast and microscopy imaging conditions for all proteins. Channel views show the whole highbit dynamic range of acquisition; contrasts were set to show colocalization of the proteins to TRIM5 $\alpha$. Blue, Hoechst; green, YFP-rhTRIM5 $\alpha$; red, anti-Rb-TRITC $(A)$, SQSTM1/p62 (B), LC3B (C), and DCP1A. (D) Number of TRIM5 $\alpha$ puncta seen and the number that colocalized with each cellular marker. (Scale bars: $20 \mu \mathrm{m}$ in overview images and $5 \mu \mathrm{m}$ in channel views.)

and annotate ribosomes and microtubules and found that they were excluded from the aggregates (Fig. 3B, red arrow, and $S I$ Appendix, Figs. S4 and S5 and Movies S1 and S7). The aggregates were not surrounded by membranes, even though other organelles were nearby. Vaults were seen embedded in the aggregates (SI Appendix, Fig. S4). Earlier correlative immunofluorescence/EM analyses of fixed and stained HeLa cells revealed the YFP-rhTRIM5 $\alpha$-associated protein p62 (26) within membranefree compartments (16), and a central aggregate-like component of the structure was termed the sequestosome. For this reason, we also call our aggregates sequestosomes.

Previous work showed that microtubules are required for TRIM5 $\alpha$ motility (11), and we found microtubules in close association with the sequestosomes in our cryo-tomograms. In some cases, the microtubules were parallel to the longer dimension of the sequestosomes, as if the sequestosomes were perhaps moving along the microtubule and being shaped by collisions with other material (Fig. 3). In many cases, the sequestosomes were also surrounded by F-actin structures (Fig. 3, SI Appendix, Fig. S5, and Movie S7) or contained these structures (SI Appendix, Fig. S6 and Movie S1). Five of these sequestosomes also appeared very close to ER tubules and mitochondria (Fig. 3 and SI Appendix, Fig. S5B, blue arrows) However, given that all cryo-tomograms were recorded in the cell periphery, it is not surprising to find cortical actin, microtubules, ER, and mitochondria. Indeed, approximately $50 \%$ of our control cryo-tomograms contained cortical actin, microtubules, ER, and mitochondria. Therefore, it may be that the sequestosomes appeared close to these structures simply by chance.

Stage 4: Phagophore Complexes. Five of the 45 YFP-rhTRIM $5 \alpha$ fluorescent bodies imaged were correlated to sequestosomes with phagophores nearby (Fig. 4, SI Appendix, Figs. S7 and S8, and Movies S3, S4, S8, and S9). We identified phagophores in our cryo-tomograms as flattened vesicles near YFP-rhTRIM5 $\alpha$ fluorescence with dimensions similar to or smaller than the sequestosomes. Only one control cryo-tomogram of HeLa cells not expressing YFP-rhTRIM5 $\alpha$ exhibited a similar flattened phagophore-like structure. The different morphologies and sizes of the phagophores visualized in our cryo-tomograms suggest they represent different stages in phagophore biogenesis. Some of our observed phagophores exhibited architecture like the omegasome structure proposed by Axe et al. (27) (Figs. 4 and 7 and SI Appendix, Fig. S8). The sequestosomes in these phagophore 

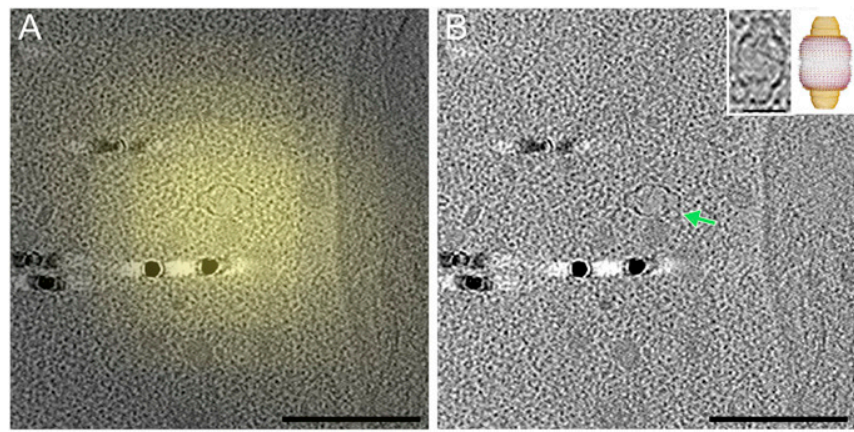

Fig. 2. Vault complexes. (A) Cryo-CLEM showing YFP-rhTRIM5 $\alpha$ fluorescent bodies localizing near vault complexes. The green arrow in $B$ highlights a vault complex. (Scale bars: $150 \mathrm{~nm}$.) (B) (Inset) Enlarged view of a vault alongside the crystal structure (Protein Data Bank [PDB] ID code 4V60) (49) of the vault complex. (Scale bar: $30 \mathrm{~nm}$.)

complexes were similar in texture and size to the prephagophore sequestosomes. Again, vaults were present, embedded within the sequestosome (SI Appendix, Figs. S7 and S8). We also observed putative phagophores without clear sequestosomes nearby (Fig. 7). All of our phagophores were in close proximity to ER, and, strikingly, in some cases the ER membrane was adjacent to a large area of the outer membrane surface of the phagophore (Figs. 4 and 7 and SI Appendix, Fig. S8). However, we did not see any ultrastructure in which the phagophore membrane is continuous with an ER subdomain or sitting between two ER cisternae in a "cradle"-like architecture $(28,29)$. However, in the two cryotomographic examples in Figs. 4 and 7, we observed four separate points of contact between the ends of the phagophores and the ER membrane, three of which were bridged by two clear stick-like densities between 16 and $21 \mathrm{~nm}$ long (Figs. $4 E$ and $F$, white double arrows and $7 G$ and $H$ ). In the cryo-tomographic example shown in Fig. 4, we also observed three 18-nm-long densities tethered between the outer surface of the phagophore and the ER membrane (Fig. $4 E-H$, red double arrows).

In three cryo-tomograms of phagophore complexes, hexagonal nets with a lattice spacing of $29 \mathrm{~nm}$ (center of one hexagon to center of the next) were observed in the cytoplasm close to the phagophore. The largest net covered a surface area of $\sim 500 \mathrm{~nm}^{2}$ and was well ordered (Fig. 7 and Movie S2). This net appeared in close proximity not only to the sequestosome, but also to mitochondria, the ER, and the ends of the phagophore membrane. The lattice spacing of this net and that formed by purified TRIM5 $\alpha$ hexamers (4) was very similar: $\sim 29 \mathrm{~nm}$ in vivo and $\sim 33 \mathrm{~nm}$ in vitro (Fig. 8). In another case, we observed a smaller hexagonal lattice inside the lumen (Fig. 4 and Movies S3 and S4). Again, the lattice spacing of this net and that formed by purified TRIM5 $\alpha$ hexamers (4) was very similar: $\sim 29 \mathrm{~nm}$ in vivo and $\sim 33 \mathrm{~nm}$ in vitro. In some cases, we also observed hexagonal lattices with a larger, $~ 35-\mathrm{nm}$ lattice spacing located on the inside surface of the plasma membrane (Fig. 3, SI Appendix, Fig. S9, black circles, and Movie S1). Based on their lattice spacing and location on the plasma membrane, which match the hexamers of triskelions that we imaged at the plasma membrane in a previous study (Fig. $8 G-L$ ) (30), we interpret these structures as clathrin. Unlike clathrin, the smaller $29-\mathrm{nm}$ nets that we observed within the YFP-rhTRIM5 $\alpha$ fluorescence signals were sandwiched between cellular material such as ribosomes and microtubules, not on the plasma membrane (Movie S2). The 29-nm nets were also not in surrounding vesicles, as would be expected for intracellular clathrin, but were rather in the cytoplasm, either unassociated with any membrane or found between ER tubules. Because their lattice spacing matches TRIM5 $\alpha$ lattices in vitro, and they colocalized with YFP-rhTRIM5 $\alpha$ fluorescence, we conclude they are YFP-rhTRIM5 $\alpha$.

Stage 5: Autophagosomes. Seven YFP-rhTRIM5 $\alpha$ fluorescent bodies colocalized to double-membraned vesicles (Fig. 5, SI Appendix, Figs. S10 and S11, and Movies S10 and S11). Only one control
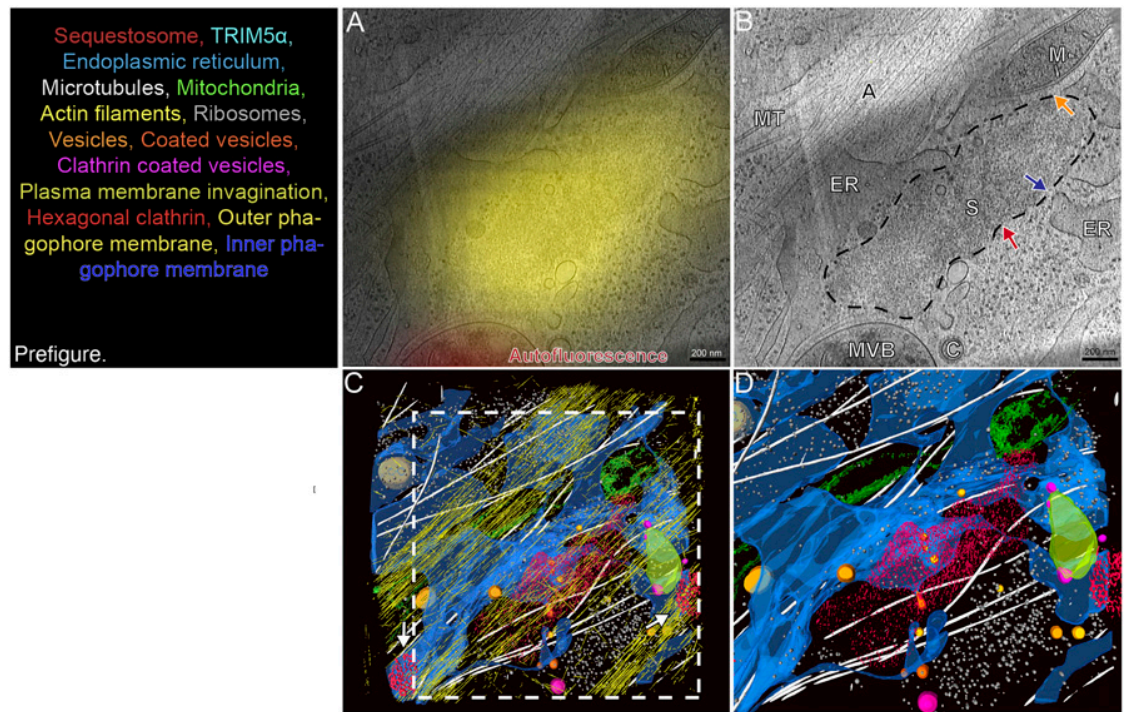

Fig. 3. Sequestosomes correlate with YFP-rhTRIM5 $\alpha$ fluorescent bodies in MG-132-treated HeLa cells. (A) Cryo-CLEM signal of YFP-rhTRIM5 $\alpha$ overlaid on a cryo-tomogram slice showing the corresponding location. (B) Same cryo-tomogram slice as in $A$, with the border of the sequestosome highlighted with a black dashed line. The red arrow highlights a cytoplasm:sequestosome boundary, the orange arrow highlights a mitochondrion:sequestosome boundary, and the blue arrow highlights ER in close proximity to the sequestosome. (Scale bars in $A$ and $B$ : $200 \mathrm{~nm}$.) (C) 3D segmentation of the features highlighted in $B$. See Prefigure for color scheme. The two white arrows point to hexagonal clathrin at the plasma membrane. (D) Enlarged view of the region indicated by the white dashed box in $C$ highlighting the ER, sequestosome, and hexagonal clathrin, with the actin filaments omitted. Here and in all subsequent figures and $S /$ Appendix figures, cellular structures are labeled as follows: ER, endoplasmic reticulum; M, mitochondrion; AV, autophagic vacuole; MVB, multivesicular body; V, vesicle; MT, microtubule; P, phagophore; A, actin filaments; F, filopodium; and AP, autophagosome. 


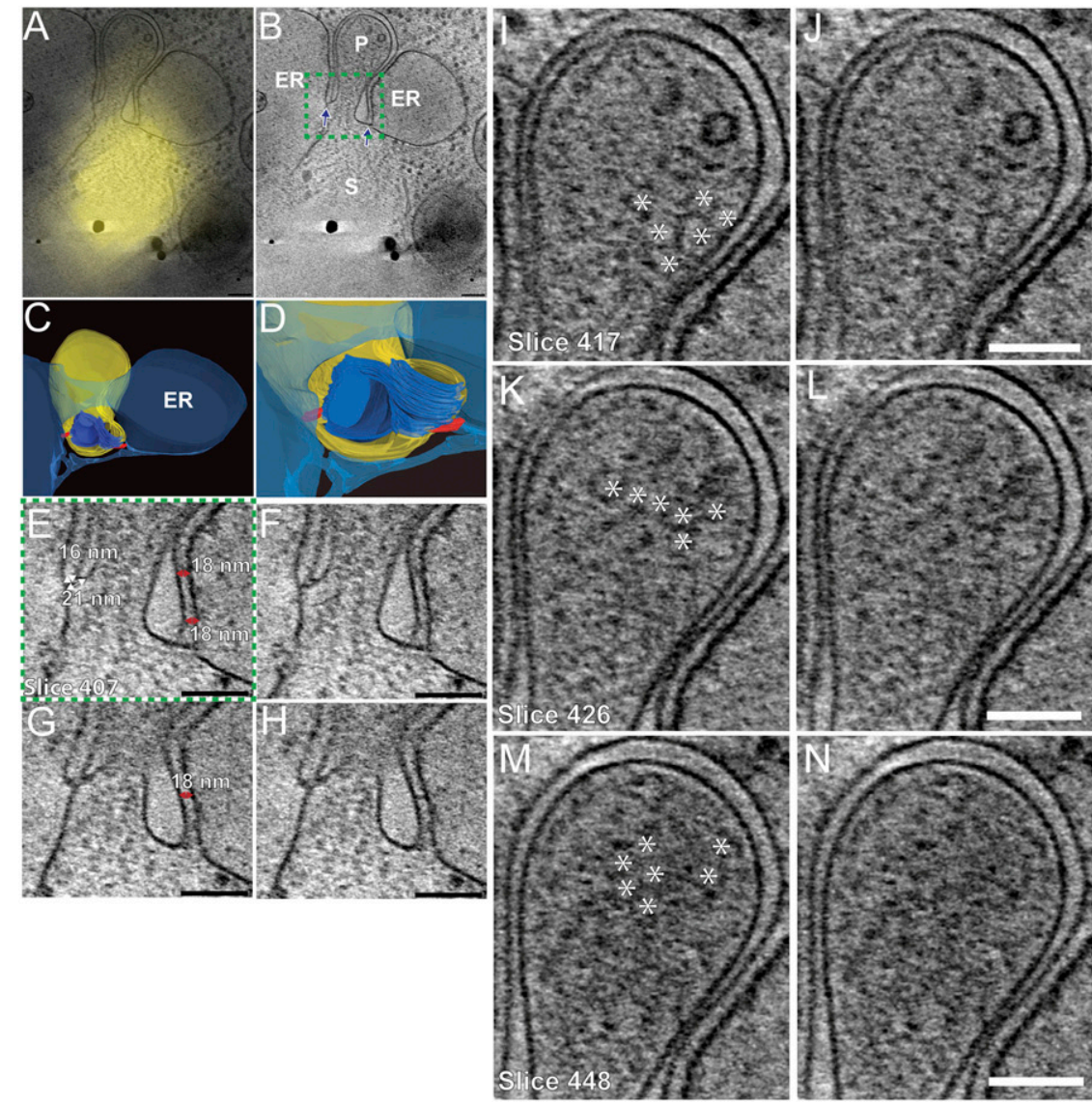

Fig. 4. Cryo-CLEM reveals YFP-rhTRIM5 $\alpha$ fluorescent bodies localized to a cytosolic sequestosome:phagophore complex in close association with the ER and a hexagonal net. $(A)$ Cryo-CLEM signal of YFP-rhTRIM5 $\alpha$ overlaid on a cryo-tomographic slice showing the corresponding location in an MG132-treated cell. (B) The same cryo-tomographic slice highlighting the phagophore and sequestosome. The blue arrows indicate the close proximity between the extreme ends of the phagophore and ER. (C) 3D segmentation of features in $B$. (D) Enlarged view of the opening of the phagophore attached to the ER membrane by tethers on either side (red isosurface). ( $E$ and $G$ ) Different cryo-tomographic slices showing enlarged views of the area indicated by the green dashed box in $B$, highlighting the opening of the phagophore attached to the ER membrane by multiple tethers (white double-headed arrows). Red arrows highlight tethers between the outer surface of the phagophore and the ER membrane. Dimensions of the densities are indicated. $(F$ and $H)$ Unlabeled views of the slices in $E$ and $G$. (Scale bars: $100 \mathrm{~nm}$.) $(I, K$, and $M$ ) Cryo-tomographic slices of the phagophore showing TRIM5 $\alpha$ hexagonal nets found inside the lumen of the phagophore. The hexagons are highlighted with asterisks to help identify the polygons in the lattice. $(J, L$, and $N$ ) Unlabeled views of the same slices. (Scale bars: $100 \mathrm{~nm}$.)

cryo-tomogram exhibited a similar structure. We interpret these as autophagosomes, organelles generated by the extension and fusion of the extreme ends of the phagophore. The autophagosomal membranes displayed variable intermembrane spaces, some with large gaps devoid of macromolecular material, such as ribosomes or microtubules (SI Appendix, Fig. S10). The texture of the material inside the autophagosomes was just like the cytoplasmic sequestosomes described earlier, except there were no filaments. Vaults were also seen inside the autophagosomes (Fig. 5).

Stage 6: Autophagic Vacuoles. In two cryo-tomograms, we saw large $(\sim 700 \mathrm{~nm})$ vesicles enclosing three or more medium-sized $(\sim 300 \mathrm{~nm})$ vesicles and various cellular debris, such as filaments and small $(\sim 40 \mathrm{~nm})$ vesicles (Fig. 6). In these two cases, the YFPrhTRIM $5 \alpha$ fluorescence localized specifically to one of the medium-sized vesicles. The interiors of these particular vesicles (that correlated with YFP-rhTRIM5 $\alpha$ ) had the same texture as the cytoplasmic sequestosomes, and they contained vaults (Fig. 6, SI Appendix, Fig. S12, and Movie S6). We interpret the large vesicles to be autolysosomes or amphisomes and the YFPrhTRIM5 $\alpha$-filled medium-sized vesicles to be remnants of autophagosomes after their outer membranes fused with the lysosome or early/late endosomes, respectively.

\section{Discussion}

Using cryo-CLEM/cryo-ET, here we found that exogenous YFPrhTRIM5 $\alpha$ colocalizes with six different stages of autophagic structures. Having made this observation, we then looked for and found substantial evidence for this in the literature (31). SI Appendix, Table S1 summarizes the numerous papers that document the association of TRIM5 $\alpha$ proteins with autophagyrelated proteins.

We found that vaults are recruited early in autophagy, and that YFP-rhTRIM5 $\alpha$ can form extended hexagonal nets in vivo. We show that intact vaults can be seen inside the autophagy pathway. We suspect that these vaults likely contain the MVP, TEP1, and the vault poly (ADP ribose) polymerase and function as specialized regulatory release compartments for exposing noncoding vault RNAs to the autophagy pathway (20).

Our results relate to several different bodies of work in the literature. First, our results support a recent report by Fletcher et al. (32) demonstrating the existence of two populations of cytoplasmic TRIM5 $\alpha$ bodies inside cells: hexagonal signaling lattices and nonsignaling aggregates. We saw both extended 

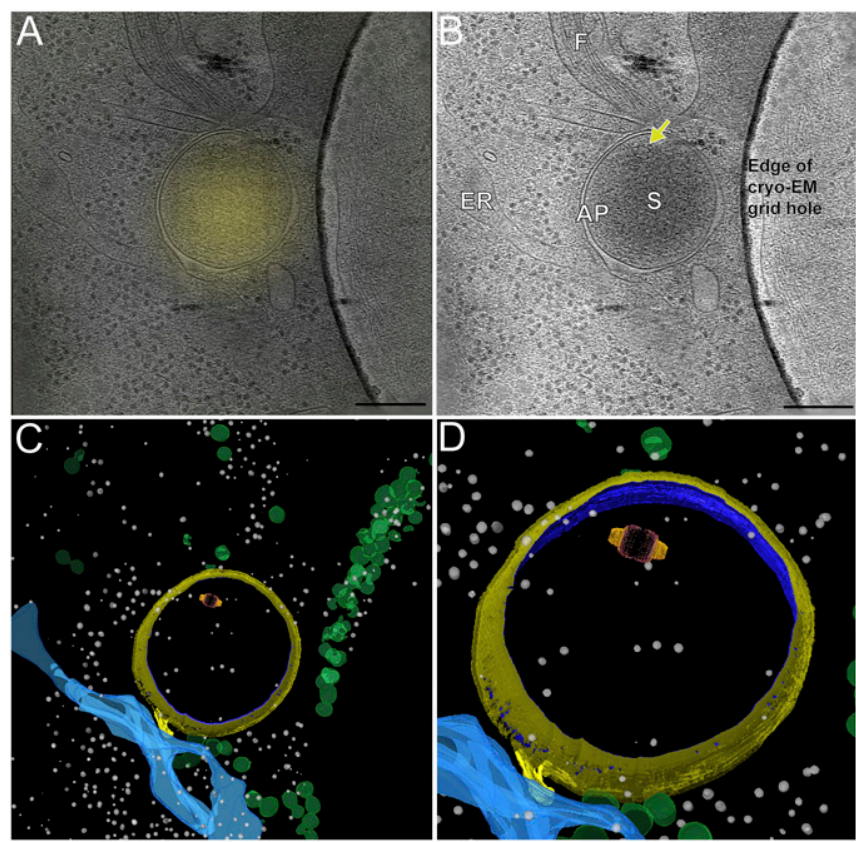

Fig. 5. Cryo-CLEM reveals YFP-rhTRIM5 $\alpha$ fluorescent bodies localized to an autophagosome containing a vault. $(A)$ Cryo-CLEM signal of YFP rhTRIM5o overlaid on a cryo-tomographic slice showing the corresponding location in an untreated cell. $(B)$ Same cryo-tomographic slice without overlaid fluorescence. Yellow arrow highlights a vault. (Scale bars: $250 \mathrm{~nm}$.) (C) 3D segmentation of features in $B$, with overlaid crystal structure of a vault (PDB ID code 4V60). (D) Enlarged view of the autophagosome shown in $C$.

hexagonal lattices and aggregates. Fletcher et al. showed that a single point mutation in the B-box domain (R119E) of huTRIM5 $\alpha$ prevented it from forming cytoplasmic fluorescent bodies or restricting retroviral infection, suggesting that preformed trimeric complexes within cytoplasmic fluorescent bodies are indispensable for viral restriction $(32,33)$. Our data support this model, because here we prove by direct imaging that TRIM5 $\alpha$ can in fact form extended hexagonal lattices inside cells.

While only three clear hexagonal nets were seen, technical limitations might have prevented us from recognizing other hexagonal nets that were present. For instance, because the nets are flexible, if the nets are too distorted, they can be difficult to recognize. Likewise, if the nets are not planar, they are difficult to recognize, because we can only look at one plane at a time on a computer screen with our eyes, and 3D segmentation does not always work well due to crowdedness and blurring in the direction of the missing wedge. Finally, the clarity of cryo-tomograms decreases with sample thickness, making nets in thick areas of the cell harder to recognize. Some of these limitations could be mitigated in the future by using thinner cell types or creating thinner samples within the cell body by cryo-focused ion beam milling.

Unfortunately, our images do not reveal what exactly the hexagonal nets are doing. Fluorescence studies have shown that overexpressed GFP-tagged human TRIM5 $\alpha$ (GFP-huTRIM5 $\alpha$ ) localizes with DFCP1 and ULK1, two early regulators of autophagy initiation, and LC3B, a key player in phagophore biogenesis $(34,35)$. A crystal structure was recently solved of the TRIM5 $\alpha$ B-box and coiled-coil regions in complex with LC3B, suggesting that this contact is specific and functional (36).

Many different receptors are used to recognize and sequester the material that is to be degraded by macroautophagy $(37,38)$. One of these receptors is the ubiquitin-binding p62 sequestosome protein, also known as SQSTM1. Interestingly, TRIM5 $\alpha$ has been shown to form complexes and to localize with p62 inside cells $(26,31,35)$. Mandell et al. recently demonstrated that $\sim 50 \%$ of TRIMs modulate autophagy not only by acting as receptors, but also by forming a molecular scaffold termed the TRIMosome $(35,39,40)$. Our localization of YFP-rhTRIM5 $\alpha$ fluorescent bodies to sites of phagophore biogenesis is in agreement with all these findings, and our finding that TRIM5 $\alpha$ can form hexagonal nets around these sites supports the notion that it may act as a scaffold. Therefore, we believe the reason we do not see hexagonal lattices in stages $1,2,3,5$, or 6 and see them only in stage 4 is because the oligomerized form of TRIM5 $\alpha$ exists only during phagophore formation, when it is active. However, we would like to point out this conclusion is still speculative, as it comes from a small number of observations from overexpressed/engineered TRIM5 $\alpha$ protein. Future studies to confirm this and to image more hexagonal lattices at sites of phagophore formation could involve using physiologically relevant levels of YFP-TRIM5 $\alpha$ with multicolor cryo-CLEM to specifically target stage 4 using colocalized YFP-TRIM5 $\alpha$ and LC3B-RFP puncta.

Concerning the aggregates, at least two kinds of aggregation compartments have been distinguished in mammalian cells: the aggresome $(41,42)$ and the aggresome-like induced structure (ALIS) $(43,44)$. Unlike our YFP-rhTRIM5 $\alpha$ aggregates, aggresomes persist in only one or two copies per cell at the microtubule organizing center near the nucleus and are surrounded by vimentin. ALIS have been shown to be formed by the aggregation of newly synthesized, ubiquitinated proteins and are induced by various cell stressors, including puromycin and oxidative stress (44). ALIS are also transient and found throughout the cell. Previous reports have shown that p62 is required for their formation (44-46), and more ALIS form when p62 is up-regulated (47). Indeed, ALIS are indistinguishable from p62 sequestosomes (45). Therefore, based on these similarities, we speculate that our YFP-TRIM5 $\alpha$ aggregates are likely ALIS rather
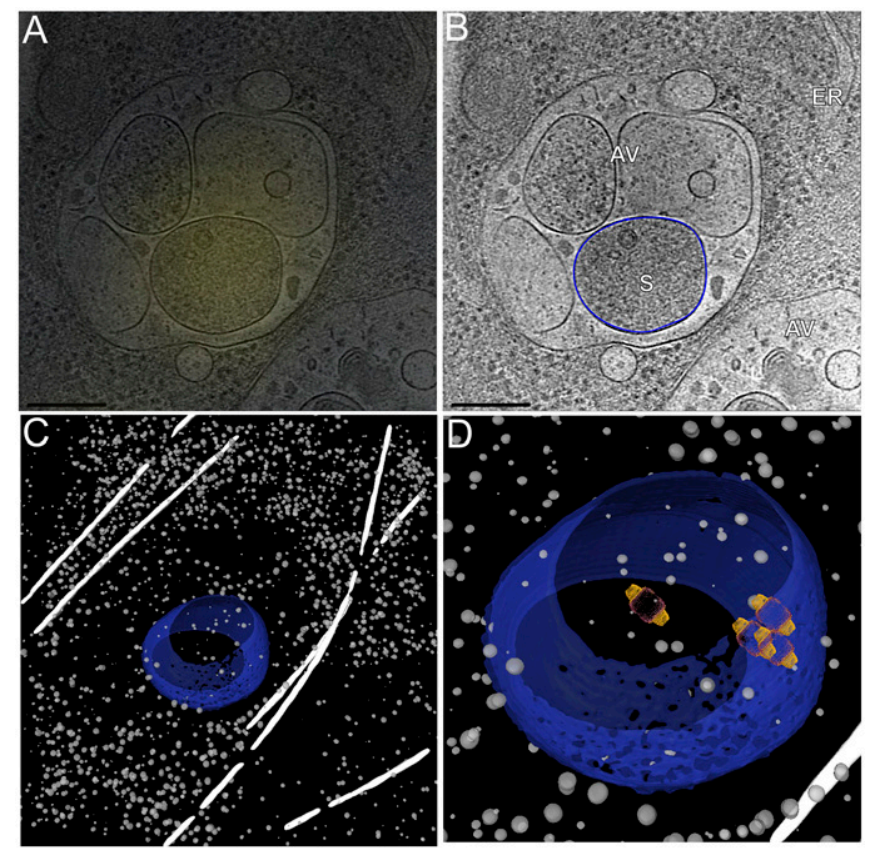

Fig. 6. Cryo-CLEM reveals YFP-rhTRIM5 $\alpha$ fluorescent bodies localized to autophagic vacuoles, and autophagic vacuoles contain vaults. $(A)$ Cryo-CLEM signal of YFP-rhTRIM5 $\alpha$ overlaid on a cryo-tomographic slice showing the corresponding location in an untreated cell. $(B)$ Same cryo-tomographic slice without overlaid fluorescence. The border of the enveloped sequestosome is highlighted with a blue line. (Scale bars: $250 \mathrm{~nm}$.) (C) 3D segmentation of features in $B$. (D) Enlarged view of the autophagic vacuole outlined in blue, with crystal structures of the vault complex (PDB ID code 4V60) overlaid. 

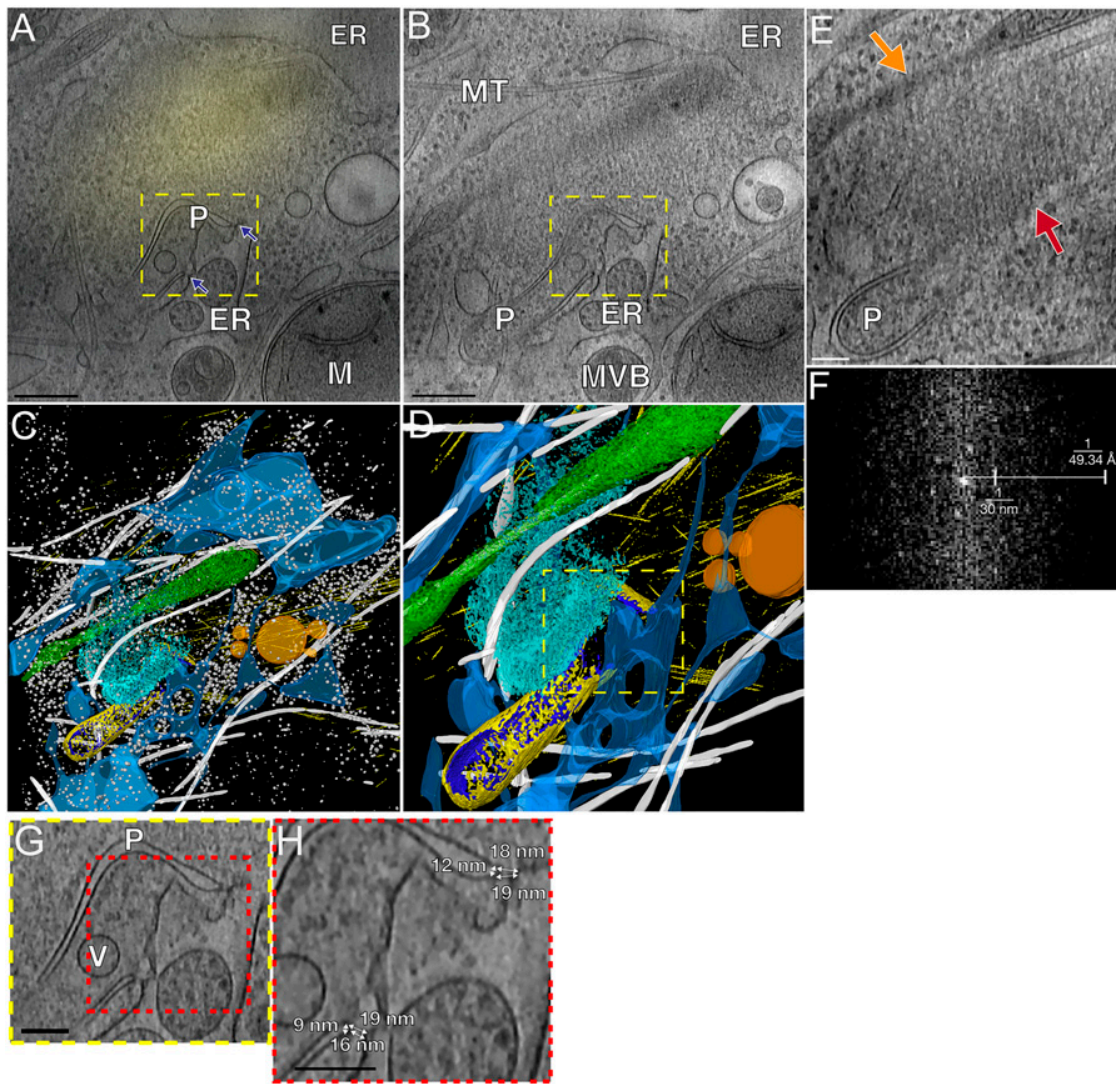

Fig. 7. Phagophores in association with a TRIM5 $\alpha$ hexagonal lattice correlate with YFP-rhTRIM5 $\alpha$ fluorescent bodies. (A) Cryo-tomographic slice displaying the base of the phagophore. The blue arrows highlight the close proximity between the extreme ends of the phagophore and the ER. (B) A different cryotomographic slice displaying the tip of the phagophore. (Scale bars in A and B: $250 \mathrm{~nm}$.) (C) 3D segmentation of the features highlighted in $B$. (D) Enlarged view of the TRIM5 $\alpha$ hexagonal lattice shown in C. (E) Cryo-tomographic slice showing a TRIM5 $\alpha$ hexagonal net located in the cytoplasm. The red arrow highlights a cytoplasm:TRIM5 $\alpha$ hexagonal net interface, and the orange arrow highlights a mitochondrion in close proximity to the TRIM5 $\alpha$ hexagonal net. (Scale bar: $100 \mathrm{~nm}$.) (F) Fourier transform of the cytosolic hexagonal TRIM5 $\alpha$. (G) Enlarged image of the area indicated by the yellow dashed boxes in $A, B$, and $D$ highlighting the extreme ends of the phagophore tethered to the ER membrane via TRIM $\alpha \alpha$ arm-like protein densities. $(H)$ Enlarged view of the region indicated by the red dashed box in $G$, highlighting the opening of the phagophore attached to the ER membrane by two tethers. Dimensions of the arm-like densities are indicated. (Scale bars: $100 \mathrm{~nm}$.)

than aggresomes. The dot-like densities in our aggregates are presumably misfolded protein, including YFP-rhTRIM5 $\alpha$ (32).

Our data contain 3D images of putative phagophores at various stages of their growth. Strikingly, we did not see ultrastructure in our cryo-tomograms like the previously described ER subdomain cradle (28). The absence of the omegasome cradle in our cryo-tomograms indicates that either we were imaging a different macroautophagy pathway or the omegasome was an artifact of traditional preparative methods (48). Future cryo-CLEM work with tagged proteins specific for omegasome formation, such as DFCP1 (27), should clarify the issue.

\section{Materials and Methods}

HeLa cells (cell line 25) were maintained in a humidified $37^{\circ} \mathrm{C}$ incubator with $5 \% \mathrm{CO}_{2}$, then cultured in DMEM medium with no phenol red (Gibco), containing $10 \% \mathrm{FBS}, 100 \mathrm{U} / \mathrm{mL}$ penicillin, and $100 \mu \mathrm{g} / \mathrm{mL}$ streptomycin. For cryoCLEM and cryo-ET, cells were plated onto fibronectin-coated 200-mesh gold R2/2 London finder Quantifoil grids (Quantifoil Micro Tools) at a density of $2 \times 10^{5}$ cells $/ \mathrm{mL}$. After a 12-h incubation, cultures were treated with MG-132 $(1 \mu \mathrm{g} / \mathrm{mL})$ for 2 to $3 \mathrm{~h}$ or left untreated, before being plunge-frozen in liquid ethane/propane mixture using an FEI Vitrobot Mark IV (21). Immediately before plunge-freezing, $3 \mu \mathrm{L}$ of a suspension of beads was applied to grids. The bead suspension was made by diluting $500 \mathrm{~nm}$ blue $(345 / 435 \mathrm{~nm})$ polystyrene fluorospheres (Phosphorex) with a colloidal solution of $20 \mathrm{~nm}$ gold fiducials (Sigma-Aldrich) pretreated with BSA. The gold served as fiducial markers for cryo-tomogram reconstruction, and the blue fluorospheres served as landmarks for registering fluorescence light microscopy
(FLM) images from different channels as well as cryo-EM images (24). Plunge-frozen grids were subsequently loaded into FEI Polara EM cartridges. EM cartridges containing frozen grids were stored in liquid nitrogen and maintained at $\leq-150{ }^{\circ} \mathrm{C}$ throughout the experiment, including cryo-FLM imaging, cryo-EM imaging, storage, and transfer.

Fluorescence Imaging and Image Processing. The EM cartridges were transferred into a cryo-FLM stage (FEI Cryostage) modified to hold Polara EM cartridges $(14,50)$ and then mounted on a Nikon Ti inverted microscope. The grids were imaged using a $60 \times$ extra-long working distance air objective (Nikon CFI S Plan Fluor ELWD 60x, NA 0.7, WD 2.62 to $1.8 \mathrm{~mm}$ ). Images were recorded using a Neo $5.5 \mathrm{sCMOS}$ camera (Andor Technology) using a two-dimensional (2D) real-time deblur deconvolution module in the NIS Elements software from AutoQuant (Nikon Instruments). The 2D real-time deconvolution algorithm estimates a point spread function using several factors, such as sample thickness, noise levels in the image, background subtraction, and contrast enhancement. All fluorescence images (individual channels) were saved in 16-bit grayscale format. YFPrhTRIM5 $\alpha$ was visualized with a YFP filter, blue fluorospheres were visualized with a DAPI filter, and red autofluorescence was imaged using an mCherry filter.

Immunofluorescence Imaging and Image Analysis. For immunofluorescence imaging cells, were plated onto fibronectin-coated 200-mesh gold R2/2 London finder Quantifoil grids (Quantifoil Micro Tools) at a density of $\sim 4 \times$ $10^{5}$ cells $/ \mathrm{mL}$ placed atop a glass slide in a $10-\mathrm{cm}$ cell culture Petri dish for $16 \mathrm{~h}$. Cells were fixed with $3 \%$ formaldehyde in PIPES buffer and washed with PBS. Grids and cells were then transferred to new glass slides, and 


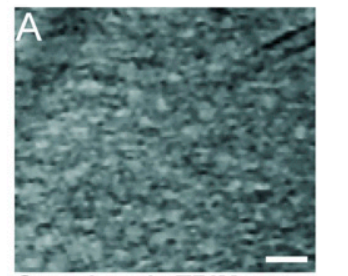

Cytoplasmic TRIM5a

(from this study)

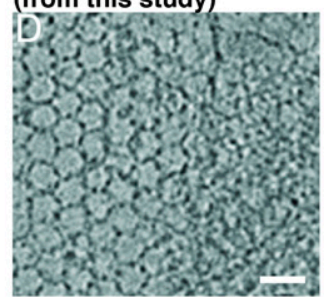

Purified TRIM5a-21R

Li et al, 2016
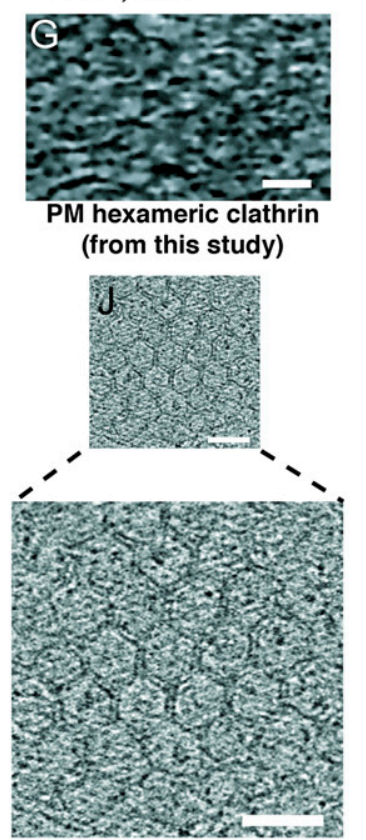

PM hexameric clathrin in adipocyte

Azubel et al, 2019

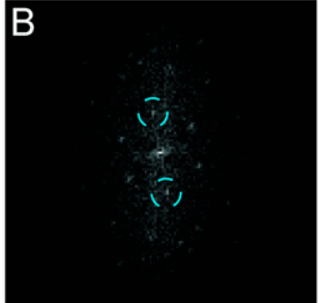

C

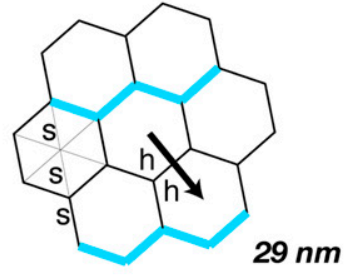

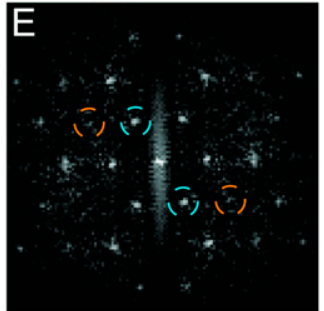

$\mathrm{F}$
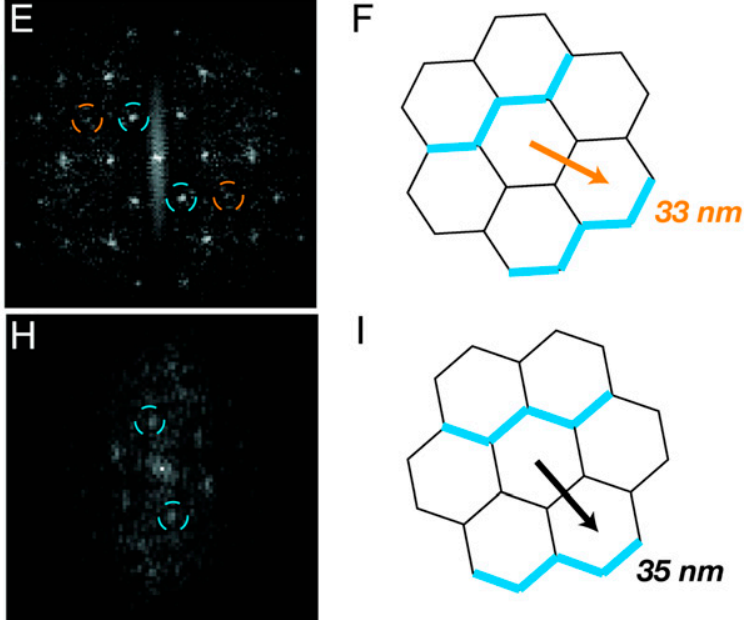

I

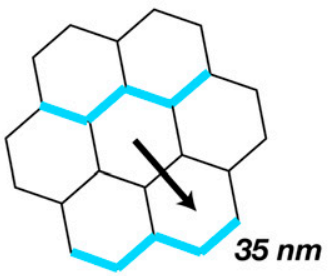

$\mathrm{L}$<smiles>CC1CC2CCC3CCC4CCC5CCC6CCC1C1C6C5C4C3C21</smiles>

Fig. 8. Hexagonal clathrin and TRIM5 $\alpha$ exhibit different lattice spacings. ( $A$ and $D$ ) Cryo-tomographic slices showing cytoplasmic hexagonal TRIM5 $\alpha$ inside a HeLa cell and a $2 D$ hexagonal lattice of purified TRIM $5 \alpha-21 R$, respectively. ( $B$ and $E$ ) Corresponding Fourier transforms. Distances between three peak pixels were measured to calculate the mean lattice spacings shown in $C$ and $F$. Details are provided in Methods. Measurement precision is $\pm 2 \mathrm{~nm}$, corresponding to the pixel size of the cryotomograms. ( $G$ and $J)$ Cryo-tomographic slices showing hexagonal clathrin inside a HeLa cell and an adipocyte cell, respectively. $(H$ and $K)$ Corresponding Fourier transforms. Distances between three peak pixels were measured to calculate the mean spacing shown in $/$ and two peak pixels for the mean spacing in $L$. (Scale bars: $50 \mathrm{~nm}$.) Blue circles in $B, E, H$, and $K$ correspond to the lowest-resolution lattice planes indicated by blue lines in $C, F, I$, and $L$. Orange circles in $E$ and $K$ correspond to the vectors indicated in $F$ and $L$. These peaks were not present in $B$ and $H$. $(J)$ Image taken from a cryo-tomogram previously recorded in the Jensen lab and described in ref. 30 .

membranes were permeabilized in a humidified container with donkey block serum with Triton-X100 surrounded by PAP pen circles of $\sim 4 \mathrm{~mm}$ for $20 \mathrm{~min}$. Incubation with primary antibodies followed for $1 \mathrm{~h}$ at room temperature in the same donkey block serum, followed by three washes in PBS and secondary anti-Rb-TRITC antibody incubation for $45 \mathrm{~min}$ at room temperature. Cells were then washed three times with PBS containing Hoechst stain. Vectashield mounting medium (VectorLabs) was added on top of the grids, followed by $\# 1.5 \mathrm{H}$ coverslip deposition over the grids for imaging.

Primary antibodies were as follows (1/100 dilution): SQSTM1/p62 (Abcam; ab109012) and LC3B clone 12k5, ZooMAb (Sigma-Aldrich; ZRK100). Controls were DCP1A antibody (Abcam; ab183709) and RAB11 (Thermo Fisher Scientific; 71-5300). Secondary antibodies (1/500 dilution) were rhodamine (TRITC) AffiniPure goat anti-rabbit IgG $(\mathrm{H}+\mathrm{L})$ (Jackson ImmunoResearch).
Images were acquired with a Nikon wide-field Ti-e2 equipped with an LED light source (Spectra $\mathrm{X}$ ) and a PCO edge 4.2BI camera using standard Chroma DAPI/GFP/TRITC/Cy5 polychroic and emission filters for DAPI/GFP/TRITC. Images were deconvolved using FlowDec (https://github.com/hammerlab/flowdec), and the Z-projections were analyzed with Icy version 2.0.3 using spots detector, colocalization studio, and HK-means plugins/modules.

EM Imaging. Cryo-EM grids previously imaged by cryo-LM were subsequently imaged by cryo-ET using an FEI G2 Polara 300 kV FEG transmission electron microscope equipped with an energy filter (slit width $20 \mathrm{eV}$ for higher magnifications; Gatan), and a 4 k $\times 4$ k K2 Summit direct detector (Gatan) in counting mode. Cryo-tomograms were also collected using an FEI Titan Krios 300-kV field emission gun transmission electron microscope equipped with a Gatan energy filter, a Gatan $\mathrm{K} 3$ direct detector in counting mode, and an FEI Volta phase plate. 
First, cellular areas containing the fluorescent bodies of interest in areas that were typically 200 to $500 \mathrm{~nm}$ thick were located in the TEM using methods described previously (24). Tilt series of these areas were then recorded using UCSF Tomography (51) or SerialEM (52) software at a mag-

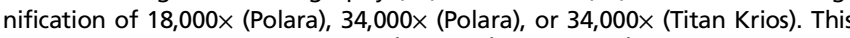
corresponds to a pixel size of $6.167 \AA, 3.260 \AA$ and $2.678 \AA$, respectively, at the specimen level and was found to be sufficient for this study. Each tilt series was collected from $-60^{\circ}$ to $+60^{\circ}$ at an increment of $1^{\circ}$ or $2^{\circ}$ in an automated fashion at 5- to 10- $\mu \mathrm{m}$ underfocus (Polara) and 3- to 5- $\mu \mathrm{m}$ underfocus (Titan Krios). The cumulative dose of one tilt series was between 80 and $200 \mathrm{e}^{-} / \AA^{2}$. The tilt series were aligned and binned by 4 into $1 \mathrm{k} \times 1 \mathrm{k}$ using the IMOD software package (53), and 3D reconstructions were calculated using the simultaneous reconstruction technique implemented in the TOMO3D software package (54) or weighted back-projection using IMOD. Noise reduction was performed using the nonlinear anisotropic diffusion method in IMOD (53), typically using a $K$ value of 0.03 to 0.04 with 10 iterations.

Fourier Transform Analysis of Hexagonal Nets. Fiji was used to measure the spacing of the atomic planes (blue lines in Fig. $8 C, F, l$, and $L$ ) corresponding to the lowest resolution peaks (blue circles) in the Fourier transform. The distance from the origin to the central pixels of three [complete net inside the cell, complete net in vitro in Li et al. (4), and clathrin imaged in this study] or two [clathrin inside the cell in Azubel et al. (30)] low-resolution peaks in each Fourier transform were measured and averaged to give a mean value. Individual values were $(28.92,28.47,28.65)$ for TRIM5 $\alpha$ inside the cell, $(32.91,32.29,32.65)$ for purified TRIM5 $\alpha-21 R$, $(33.95,35.15,34.89)$ for clathrin inside a HeLa cell, and (34.19 and 35.09) for clathrin inside an adipocyte cell. If the hexagonal lattice is partitioned into triangles, as shown in Fig. $5 C$, then this distance between the

1. A. Reymond et al., The tripartite motif family identifies cell compartments. $E M B O \mathrm{~J}$. 20, 2140-2151 (2001).

2. M. Stremlau et al., The cytoplasmic body component TRIM5alpha restricts HIV-1 infection in Old World monkeys. Nature 427, 848-853 (2004).

3. B. K. Ganser-Pornillos et al., Hexagonal assembly of a restricting TRIM $5 \alpha$ protein. Proc Natl. Acad. Sci. U.S.A. 108, 534-539 (2011).

4. Y.-L. Li et al., Primate TRIM5 proteins form hexagonal nets on HIV-1 capsids. eLife 5, e16269 (2016)

5. J. G. Sanchez et al., The tripartite motif coiled-coil is an elongated antiparallel hairpin dimer. Proc. Natl. Acad. Sci. U.S.A. 111, 2494-2499 (2014).

6. J. M. Wagner et al., Mechanism of B-box 2 domain-mediated higher-order assembly of the retroviral restriction factor TRIM5 $\alpha$. eLife 5, e16309 (2016).

7. S. Nisole, C. Lynch, J. P. Stoye, M. W. A. Yap, A Trim5-cyclophilin A fusion protein found in owl monkey kidney cells can restrict HIV-1. Proc. Natl. Acad. Sci. U.S.A. 101 13324-13328 (2004)

8. D. M. Sayah, E. Sokolskaja, L. Berthoux, J. Luban, Cyclophilin A retrotransposition into TRIM5 explains owl monkey resistance to HIV-1. Nature 430, 569-573 (2004).

9. M. Stremlau, M. Perron, S. Welikala, J. Sodroski, Species-specific variation in the B30.2(SPRY) domain of TRIM5alpha determines the potency of human immunodeficiency virus restriction. J. Virol. 79, 3139-3145 (2005).

10. M. Stremlau et al., Specific recognition and accelerated uncoating of retroviral capsids by the TRIM5alpha restriction factor. Proc. Natl. Acad. Sci. U.S.A. 103, 5514-5519 (2006).

11. E. M. Campbell et al., TRIM5 alpha cytoplasmic bodies are highly dynamic structures. Mol. Biol. Cell 18, 2102-2111 (2007)

12. E. M. Campbell, O. Perez, J. L. Anderson, T. J. Hope, Visualization of a proteasomeindependent intermediate during restriction of HIV-1 by rhesus TRIM $5 \alpha$. J. Cell Biol. 180, 549-561 (2008)

13. K. Narayan et al., Multi-resolution correlative focused ion beam scanning electron microscopy: Applications to cell biology. J. Struct. Biol. 185, 278-284 (2014).

14. A. Briegel et al., "Chapter thirteen-Correlated light and electron cryo-microscopy" in Methods in Enzymology, G. J. Jensen, Ed. (Academic Press, 2010), Vol. 481, pp. 317-341.

15. K. R. Parzych, D. J. Klionsky, An overview of autophagy: Morphology, mechanism, and regulation. Antioxid. Redox Signal. 20, 460-473 (2014).

16. G. Bjørkøy et al., p62/SQSTM1 forms protein aggregates degraded by autophagy and has a protective effect on huntingtin-induced cell death. J. Cell Biol. 171, 603-614 (2005).

17. V. Rogov, V. Dötsch, T. Johansen, V. Kirkin, Interactions between autophagy receptors and ubiquitin-like proteins form the molecular basis for selective autophagy. Mol. Cell 53, 167-178 (2014).

18. A. Stolz, A. Ernst, I. Dikic, Cargo recognition and trafficking in selective autophagy Nat. Cell Biol. 16, 495-501 (2014).

19. C. L. Woodward, L. M. Mendonça, G. J. Jensen, Direct visualization of vaults within intact cells by electron cryo-tomography. Cell. Mol. Life Sci. CMLS 72, 3401-3409 (2015).

20. R. Horos et al., The small non-coding vault RNA1-1 acts as a riboregulator of autophagy. Cell 176, 1054-1067.e12 (2019).

21. C. V. lancu et al., Electron cryotomography sample preparation using the Vitrobot. Nat. Protoc. 1, 2813-2819 (2006) lowest-resolution planes corresponds to $3 \mathrm{~s}$. The distance between the centers of two adjacent hexagons ( $2 \mathrm{~h})$ can then be calculated as follows:

$$
\begin{aligned}
\frac{h}{s}=\cos 30^{\circ} & =\sqrt{3} / 2=0.866 \\
2 h & =\sqrt{3} \mathrm{~s}
\end{aligned}
$$

These distances, rounded to the nearest integer, are reported in Fig. 8.

Segmentation and Isosurface Generation. Segmentation and isosurface rendering were performed in Amira (FEI). The ER, phagophores, autophagosomes, autophagic vacuoles, mitochondria, and vesicles were segmented manually using the thresholding tool. The actin filaments were segmented using the Amira XTracing Extension in Amira $(55,56)$. The microtubules and ribosomes were segmented using the tomoseg machine learning module in EMAN2 $(57,58)$. Movie image sequences were generated in JPEG format in Amira (FEI) and IMOD, then converted into movies using QuickTime Player 7. Adobe Photoshop CS6 was then used to produce the final versions of the movies.

Data Availability. All study data are included in the article and SI Appendix.

ACKNOWLEDGMENTS. We thank Sarah Speed (Division of Biology and Biological Engineering, California Institute of Technology) for valuable help in segmenting cryo-tomograms and Catherine Oikonomou (Division of Biology and Biological Engineering, California Institute of Technology) for insights and comments on the manuscript. This work was supported in part by NIH Grant AI150464 (to G.J.J. and T.J.H.).

22. X. Wu, J. L. Anderson, E. M. Campbell, A. M. Joseph, T. J. Hope, Proteasome inhibitors uncouple rhesus TRIM5 $\alpha$ restriction of HIV-1 reverse transcription and infection. Proc. Natl. Acad. Sci. U.S.A. 103, 7465-7470 (2006)

23. S. D. Carter et al., Ribosome-associated vesicles: A dynamic subcompartment of the endoplasmic reticulum in secretory cells. Sci. Adv. 6, eaay9572 (2020).

24. S. D. Carter et al., Distinguishing signal from autofluorescence in cryogenic correlated light and electron microscopy of mammalian cells. J. Struct. Biol. 201, 15-25 (2018).

25. F. Reggiori, C. Ungermann, Autophagosome maturation and fusion. J. Mol. Biol. 429, 486-496 (2017)

26. C. O'Connor et al., p62/sequestosome-1 associates with and sustains the expression of retroviral restriction factor TRIM5 $\alpha$. J. Virol. 84, 5997-6006 (2010).

27. E. L. Axe et al., Autophagosome formation from membrane compartments enriched in phosphatidylinositol 3-phosphate and dynamically connected to the endoplasmic reticulum. J. Cell Biol. 182, 685-701 (2008).

28. M. Hayashi-Nishino et al., A subdomain of the endoplasmic reticulum forms a cradle for autophagosome formation. Nat. Cell Biol. 11, 1433-1437 (2009).

29. P. Ylä-Anttila, H. Vihinen, E. Jokitalo, E.-L. Eskelinen, 3D tomography reveals connections between the phagophore and endoplasmic reticulum. Autophagy 5, 1180-1185 (2009).

30. M. Azubel et al., FGF21 trafficking in intact human cells revealed by cryo-electron tomography with gold nanoparticles. eLife 8, e43146 (2019).

31. C. M. S. Ribeiro et al., Receptor usage dictates HIV-1 restriction by human TRIM $5 \alpha$ in dendritic cell subsets. Nature 540, 448-452 (2016).

32. A. J. Fletcher et al., Trivalent RING assembly on retroviral capsids activates TRIM5 ubiquitination and innate immune signaling. Cell Host Microbe 24, 761-775.e6 (2018)

33. F. Diaz-Griffero et al., Modulation of retroviral restriction and proteasome inhibitor resistant turnover by changes in the TRIM5 $\alpha$ B-box 2 domain. J. Virol. 81, 10362-10378 (2007).

34. E. Itakura, N. Mizushima, Characterization of autophagosome formation site by a hierarchical analysis of mammalian Atg proteins. Autophagy 6, 764-776 (2010).

35. M. A. Mandell et al., TRIM proteins regulate autophagy and can target autophagic substrates by direct recognition. Dev. Cell 30, 394-409 (2014).

36. J. R. Keown et al., A helical LC3-interacting region mediates the interaction between the retroviral restriction factor Trim $5 \alpha$ and mammalian autophagy-related ATG8 proteins. J. Biol. Chem. 293, 18378-18386 (2018).

37. J. Kaur, J. Debnath, Autophagy at the crossroads of catabolism and anabolism. Nat. Rev. Mol. Cell Biol. 16, 461-472 (2015).

38. M. L. Seibenhener et al., Sequestosome $1 / \mathrm{p} 62$ is a polyubiquitin chain binding protein involved in ubiquitin proteasome degradation. Mol. Cell. Biol. 24, 8055-8068 (2004).

39. M. A. Mandell, T. Kimura, A. Jain, T. Johansen, V. Deretic, TRIM proteins regulate autophagy: TRIM5 is a selective autophagy receptor mediating HIV-1 restriction Autophagy 10, 2387-2388 (2014)

40. M. A. Mandell et al., TRIM17 contributes to autophagy of midbodies while actively sparing other targets from degradation. J. Cell Sci. 129, 3562-3573 (2016).

41. R. García-Mata, Z. Bebök, E. J. Sorscher, E. S. Sztul, Characterization and dynamics of aggresome formation by a cytosolic GFP-chimera. J. Cell Biol. 146, 1239-1254 (1999)

42. J. A. Johnston, C. L. Ward, R. R. Kopito, Aggresomes: A cellular response to misfolded proteins. J. Cell Biol. 143, 1883-1898 (1998).

43. H. Lelouard et al., Transient aggregation of ubiquitinated proteins during dendritic cell maturation. Nature 417, 177-182 (2002) 
44. J. Szeto et al., ALIS are stress-induced protein storage compartments for substrates of the proteasome and autophagy. Autophagy 2, 189-199 (2006).

45. T. H. Clausen et al., p62/SQSTM1 and ALFY interact to facilitate the formation of p62 bodies/ALIS and their degradation by autophagy. Autophagy 6, 330-344 (2010)

46. S. Pankiv et al., p62/SQSTM1 binds directly to Atg8/LC3 to facilitate degradation of ubiquitinated protein aggregates by autophagy. J. Biol. Chem. 282, 24131-24145 (2007).

47. X.-D. Liu et al., Transient aggregation of ubiquitinated proteins is a cytosolic unfolded protein response to inflammation and endoplasmic reticulum stress. J. Biol. Chem. 287, 19687-19698 (2012).

48. M. Pilhofer, M. S. Ladinsky, A. W. McDowall, G. J. Jensen, "Bacterial TEM: New insights from cryo-microscopy" in Methods in Cell Biology, T. Müller-Reichert, Ed. (Academic Press, 2010), vol. 96, chap. 2, pp. 21-45.

49. H. Tanaka et al., The structure of rat liver vault at 3.5 angstrom resolution. Science 323, 384-388 (2009)

50. S. Nickell, C. Kofler, A. P. Leis, W. Baumeister, A visual approach to proteomics. Nat Rev. Mol. Cell Biol. 7, 225-230 (2006).
51. S. Q. Zheng et al., UCSF tomography: An integrated software suite for real-time electron microscopic tomographic data collection, alignment, and reconstruction. J. Struct. Biol. 157, 138-147 (2007).

52. D. N. Mastronarde, Automated electron microscope tomography using robust prediction of specimen movements. J. Struct. Biol. 152, 36-51 (2005).

53. J. R. Kremer, D. N. Mastronarde, J. R. McIntosh, Computer visualization of threedimensional image data using IMOD. J. Struct. Biol. 116, 71-76 (1996).

54. J. I. Agulleiro, J. J. Fernandez, Fast tomographic reconstruction on multicore computers. Bioinformatics 27, 582-583 (2011).

55. A. Rigort et al., Automated segmentation of electron tomograms for a quantitative description of actin filament networks. J. Struct. Biol. 177, 135-144 (2012).

56. B. Weber et al., Automated tracing of microtubules in electron tomograms of plastic embedded samples of Caenorhabditis elegans embryos. J. Struct. Biol. 178, 129-138 (2012).

57. M. Chen et al., Convolutional neural networks for automated annotation of cellular cryo-electron tomograms. Nat. Methods 14, 983-985 (2017).

58. M. Chen et al., Protocol for convolutional neural networks based automated cellular cryo-electron tomograms annotation. Protoc. Exch., 10.1038/protex.2017. 095 (2017). 